\title{
Research Paper: Interference of Various Sources of Sensory Feedback on Transition of Relative Phase in Bimanual Coordination on Active and Inactive Wom- an With Multiple Sclerosis
}

\author{
Ebrahim Norouzi $^{\mathbf{1}^{*}}$ \\ 1. Department of Motor Behavior and Sport Management, Faculty of Sport Sciences, Urmia University, Urmia, Iran.
}

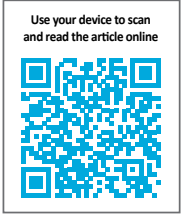

Citation: Norouzi E. Interference of Various Sources of Sensory Feedback on Transition of Relative Phase in Bimanual Coordination on Active and Inactive Woman With Multiple Sclerosis. Physical Treatment. 2017; 6(4):201-210. https://doi. org/10.18869/NRIP.PTJ.6.4.201

https://doi.org/10.18869/NRIP.PTJ.6.4.201

Article info:

Received: 02 Aug. 2016 Accepted: 17 Nov. 2016

Keywords:

Relative phase transition, Multiple Sclerosis, Bimanual coordination, Sensory feedback

\begin{abstract}
A B S T R A C T
Purpose: Comparing the effects of manipulating senses on relative phase transition bimanual coordination pattern of active and inactive women with Multiple Sclerosis (MS).

Methods: The methodology of this study was repeated-measures design. Study participants comprised 10 active women and 10 inactive women with multiple sclerosis who voluntarily participated in this experiment. The participants ( $\mathrm{n}=20$; aged $18-25$ years) performed bimanual in-phase and anti-phase movements with their wrists at three different speeds ranging from slow to fast and 5 different sensory conditions, including 1) normal sensory input, 2) masked vision, 3) masked proprioception, 4) masked audition, and 5) full sensory deprivation. Two-way (5 sensory conditions $\times 2$ groups) analyses of variance for repeated measures (ANOVA) were performed using SPSS 24.

Results: The findings showed that the main effect of the senses and groups was not significant, while the effect of interaction between the senses and groups was significant $(\mathrm{P}=0.047)$. Accordingly, there was a significant difference between two groups with regard to the manipulation of proprioception, vision, and audition conditions. Regarding the effect of different senses in both active and inactive women with MS, only a significant effect was observed between two groups in manipulation of proprioception condition, when vision and audition were not manipulated $(\mathrm{P}=0.004)$. Also, comparing active and inactive woman with MS in different manipulated sensory conditions did not reveal significant differences.
\end{abstract}

Conclusion: Proprioception in both active and inactive woman with MS has a significant impact on performing bimanual coordination task.

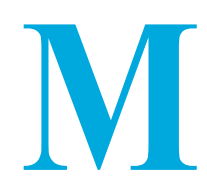

\section{Introduction}

any daily activities require continuous updating of the perception-action cycle to maintain the accuracy of hu- man motor behavior [1]. Among the different sensory modalities, vision, proprioception, and audition have an essential role in planning and guiding movements in time and space [2-4]. Daily motor tasks often demand

\section{* Corresponding Author:}

Ebrahim Norouzi, PhD Candidate

Address: Department of Motor Behavior and Sport Management, Faculty of Sport Sciences, Urmia University, Urmia, Iran.

Phone: +98 (912) 9469087

E-mail: ebrahim.norouzi68@gmail.com 
using our hands simultaneously rather than separately, necessitating performance of bimanual movements [5].

For upper limb movements made in the horizontal plane, these principles of coordination are realized in two stable patterns: in-phase ( 00 relative phase) and anti-phase (1800 relative phase). The in-phase coordination mode refers to mirror-symmetrical movements made simultaneously towards and away from the body midline and involves concurrent activation of homologous muscle groups [6-9]. The anti-phase coordination mode refers to movements made, simultaneously, in the same direction from one side of body midline to its other side; resulting in performance of parallel (isodirectional) movement pattern in extrinsic space [6, 10-12]. With increasing movement frequency, in-phase coordination remains stable while anti-phase coordination destabilizes, and if unopposed, eventually results in a spontaneous transition to the in-phase movement pattern $[7,13,14]$.

It is generally acknowledged that sensory feedback plays a dominant role in fine-tune movement control. Sensory input for feedback control may include proprioception, vision, and audition. Vision is often regarded as the most important perceptive modality during interaction with environment in daily life. At least for perceiving spatial information, vision dominates other senses [15]. Many motor tasks are impossible, or at least, are much harder to perform without vision, for example, walking on an uneven terrain, hitting a tennis ball, or skiing [15]. Confirmed evidence on the critical role of vision in the coupling of limb movements came from studies using both discrete [16] and cyclical bimanual movements [17]. Specifically, these studies showed that bimanual movements were performed with higher levels of accuracy and stability when visual information on the position of the moving effectors was available as compared to other conditions where visual feedback was absent. Vision, which provides information about target and hand positions, is generally considered to be the main cue leading to sensory motor adaptation, whereas proprioception is thought to be secondary [18-20].

Besides vision, proprioception is also an important source of feedback which is essential for maintaining the required coordination patterns during bimanual movements [16]. Proprioceptive input from the muscle spindles and tendons is crucial for movement control. It allows the central nervous system to monitor the position and speed of the moving limbs and adjust the motor command if necessary. The coordination of ongoing movements uses proprioception in healthy participants $[21,22]$, while deafferentiated patients exhibit clear co- ordination deficits [23, 24]. Proprioception, however, cannot fully account for successful performance of a coordination task. For example, coordination deficits in deafferentiated patients become apparent only if vision is absent [5, 23-25]. The relative contribution of vision and proprioception to the control of a coordinated movements may depend, nonetheless, on the nature of the task. For example, there is evidence to show that a bimanual circle-drawing task where movements always continue in the same direction with no reversal movements is controlled by proprioceptive feedback [26]. On the other hand, control of bimanual coordination tasks in which the effectors must stop and reverse direction, entails reliance on the use of vision and proprioception [8].

Another source for sensory feedback which plays an important role in the regulation of coordinated behavior is audition. For instance, top performance in table tennis requires auditory information about the ball bouncing on the table and racket [27]. Perhaps audition could also be taken as a sensory input influencing the coupling of the limbs during bimanual coordination as the sounds produced by the motion may provide feedback about performance. In fact, the association between the auditory and the motor systems has been examined in a recent study during performance of bimanual linear coordination task with a sliding device [28]. Yet, findings from this study suggested that the absence of auditory feedback from the motion of the sliding device was not essential for timing of the coordination patterns. Also auditory is an important source of sensory information for people with visual impairment [29].

Loss of hearing is one of the rare signs in patients with Multiple Sclerosis (MS) and about $6 \%$ of patients with MS are experiencing an auditory disorder. Hearing loss is associated with other symptoms such as vision problems, lack of balance control commonly in patients with MS that may be created because of damage in the central nervous system (brain stem). It is believed that hearing loss in patients with MS, is due to the injury and inflammation around the nerve center of the eighth that is the location of the auditory nerve [30]. MS is a disease, which unfortunately has affected many young boys and girls [30] in the present century. The most common age of starting this disease is the young age and approximately two times more prevalent in women than men [31]. Each movement from simple to complex, requires coordination of all parts of the body. Meanwhile the patients with MS because have decreased motor performance compared to healthy subjects and many researchers and physicians are trying to increase the motor performance and motor coordination in these patients. 
In the present study, we used a bimanual coordination task consisting of flexion and extension movements with both wrists in either an in-phase or an anti-phase mode. The continuous nature of the bimanual actions in the present study requires the participants to control the limb extensively in an online manner, through visual, proprioception, or audition feedback loops. Assessing the relative phase transitions contribution of each aforementioned sensory source on the strength of coupling between the two wrists in active and inactive woman with MS was our primary goal. It is also of our interest to further insight into the dynamics of bimanual coordination by examining how deprivation of the three sensory sources would affect performance. It is generally acknowledged that one requires proprioceptive and visual information to fine tune motor patterns. Exploring the coordinative behavior at the absence of all sensory sources received less investigation, especially in patients with MS. However, to our knowledge, the technique of sensory feedback transformations has been used mainly in unilateral tasks whereas bimanual tasks have received much less attention. The present experimental design also addressed the question whether in-phase and anti-phase bimanual coordination patterns (which vary from each other by the degrees of their performance stability at increasing movement speeds) are differentially affected by the absence or presence of visual, proprioceptive and or auditory feedbacks in active and inactive woman with MS.

\section{Materials and Methods}

\section{Participants}

The design of this study was quasi-experimental. The statistical population consisted of all active and inactive patients with MS in Tehran City; of them 20 young females were selected by convenience sampling method. The criteria for selection of active patients consist of ability to carry out routine tasks and do exercise three times a week. The study participants were female patients with MS and EDSS (Expanded Disability Status Scale) score between 2 and 5.5 who were referred to MS Society Rehabilitation Clinic of Dr. Shah Begay. All participants were right handed (assessed by the Edinburgh Handedness Inventory) [32] adults, aged 18-26 years with a mean age of 21 years. Also, all had normal vision based on the Snellen chart test. Participants were also asked to complete questionnaires on their health prior to their inclusion in the study. All participants read and signed an informed consent form, which has been approved by the local Ethics Board (Urmia University).

\section{Apparatus}

Participants sat on an adjustable chair at a table covered by a white laminated poster board $(50 \mathrm{~cm}$ deep and $86 \mathrm{~cm}$ wide). Wrist movements were permitted in only the extension and flexion orientation from midline. Attached in parallel to the slides were linear potentiometers (Bourns Instruments, Riverside, CA), which encoded the displacement of the handled over a 20-second trial. Data were sampled using a microprocessor (80486) with a sampling rate of $150 \mathrm{~Hz}$. Lab Windows software (National instrument corporation, version 2.2.1) initiated and terminated 20 -second trials and also provided data capture and recording of limb position over time.

An auditory metronome (NCH Swift Sound Tone Generator, version 2.01) provided pacing information for bimanual task [28]. In auditory deprivation condition, a with-noise masking stimulus (NCH Swift Sound Tone Generator, version 2.01) was delivered to the subjects' ears via supra- aural headphones (optimum pro 155 stereo headphone) so that audition about performance from the linear wrist task was masked. We used a self-build tendon vibrator consisted of pager and small vibration motors, which rotated an unbalanced mass attached to the shaft of a small magnet DC motor. This apparatus constitutes very low-cost actuators for inducing tendon vibration. We have also used a Panasonic vibration motor (micro-motor with dimensions $0.59 \times 1.15$ ) with an operating range of $150 \mathrm{~Hz}$. It was reported by Naito et al. [33] that although some qualitative aspects of the illusion were affected by the amplitude of vibration, illusion strength was determined mainly by vibration frequency. The surface area of the vibrator head was adjusted to allow an optimal contact with the skin by adding bars with different profiles. The vibrator was positioned over the wrist tendon near the radiocarpal joint and vibration frequency was set for each participant to generate maximum illusion.

\section{Procedure}

The participants were willing to do the task. They were asked to take two handles adhered to the moving slides and put them horizontally in the left-right dimension (wrist extension and flexion). While grasping two handles, the participants produced 00 relative phase (inphase) and 1800 relative phase (anti-phase) patterns. In the in-phase patterns, the participants were instructed to move their hands toward and then away from each other symmetrically and continuously by activating homologous flexor and extensor muscles of the wrists. In the anti-phase patterns, the limbs moved together in an isodirectional fashion with homologous muscle group 
contracting in an alternating fashion. The participants received instructions to keep pace with a metronome by performing a complete cycle of in-out-in handle altering in time with the beat. The metronome outpaced the assigned speed or frequency of limb movement starting bat a slow speed equivalent to a frequency of 58 beat in minute for 20 seconds. After completion of the 20 -second trial at slow speed, the same required coordination task was paced at a medium metronome frequency $(90$ beat per minute), and subsequently at a fast metronome frequency ( 152 beat per minute).

When the participants entered the laboratory, we obtained consent (and assent, when appropriate). Next, we conducted a handedness inventory after the participants were seated. We encouraged the participants to perform in-phase and anti-phase patterns during experiment. There were 5 counterbalanced conditions: 1) normal sensory condition (vision, proprioception, and audition were available); 2) masked vision (normal proprioception and audition); 3) masked proprioception by tendon vibration (normal vision and audition); 4) masked audition by white noise (normal vision and proprioception); and 5) full sensory deprivation (no vision, masked proprioception, and masked audition).

\section{Data processing}

The position signals were smoothed with a symmetrical Bartlett (triangular) filter. Velocity time series were derived from the position signal using a 2-point central difference algorithm and then smoothed with a Bartlett window. The smoothed position and velocity time series were then used to calculate each component of the near-continuous phase state for each trial according to the formula:

$$
\phi R=\tan -1\{(d X R / d t) / X R\}
$$

, where $\phi R$ is the phase of the right wrist at each sample, $\mathrm{XR}$ is the position of the right wrist rescaled to the interval $\{-1,1\}$ for each cycle of oscillation, and $(\mathrm{dXR} / \mathrm{dt})$ is its normalized instantaneous velocity. The same formula was used to calculate from the position and velocity signals of the left wrist. The relative phase $(\varphi)$ between the two wrists, was then expressed as:

$$
\varphi=\phi R-\phi L
$$

The mean absolute error of relative phase (AE) reflected the deviation from the target relative phase $\left(0^{\circ}\right.$ for the in-phase mode and $180^{\circ}$ for the anti-phase mode) ('coordination accuracy'). The standard deviation of relative phase $(S D \varphi)$ referred to the spread of relative phase measures around the mean (coordination consistency).

\section{Statistics}

$A E \varphi$ and $S D \varphi$ scores were determined for each trial and subsequently averaged across test trials of each condition. Two-way ( 5 sensory conditions $\times 2$ groups) analyses of variance for repeated measures (ANOVA) were implemented by using Statistical software (version 8.0). The significant results of interest were investigated post hoc by using pairwise comparisons with Bonferroni correction. The level of significance was set to $\mathrm{P}=0.05$.

\section{Results}

Sensory condition and group on coordination consistency

The main effect for group $(\mathrm{P}=0.123)$ and sensory condition $(\mathrm{P}=0.156)$ did not reach the level of significance. However, interactions between sensory conditions and groups for the $\mathrm{AE}$ scores were visible in relative phase transition (Table 1). The results of the $5 \times 2$ ANOVA for the relative phase transition revealed a significant interaction effect for sensory condition and group ( $F 4,56=325.13, \mathrm{P}=0.001)$. This issue showed a significant difference between two groups in sensory condition of the vision, audition, and proprioception masked. To study the mutual impact, a 2-way analysis of variance test was utilized to investigate different sensory conditions between two groups (Figure 1). Moreover, 2-way replicated measures analysis of variance tests was done to assess differences between each experimental condition in either group separately. In order to do so, the adjustment coefficient alpha was used. Table 2 presents the results of follow-up. As shown in Table 2, the only significant effect was observed between the two groups in a state of proprioception sense manipulated along with normal vision and audition $(\mathrm{P}=0.004)$. Figure 2 shows situation of the trial between the two groups.

Figure 2 shows that in every sensory condition, MS active group have been better than MS inactive one. Only in the full sensory deprivation condition, MS inactive group slightly performed better than MS active group.

\section{Discussion}

The experiment was designed to investigate whether sensory information contributed by proprioception, vision, and audition is parametrically redundant or distinct. MS active and inactive subjects performed coordination 
Table 1. The results of analysis of variance with repeated measures to investigate the relative phase transition between active and inactive groups with MS

\begin{tabular}{rcccccc}
\hline Statistical Indicators & Sum of Squares & df & Mean Square & F & Sig. & Etta Square \\
\hline Source & 6.398 & 4 & 599.1 & 713.1 & 0.156 & 0.087 \\
Sensory & 498.9 & 4 & 374.2 & 543.2 & 0.047 & 0.124 \\
Sensory group & 986.7 & 1 & 986.7 & 625.2 & 0.123 & 0.127 \\
Group & & & & & PHYSICAL TREA TMENTS
\end{tabular}

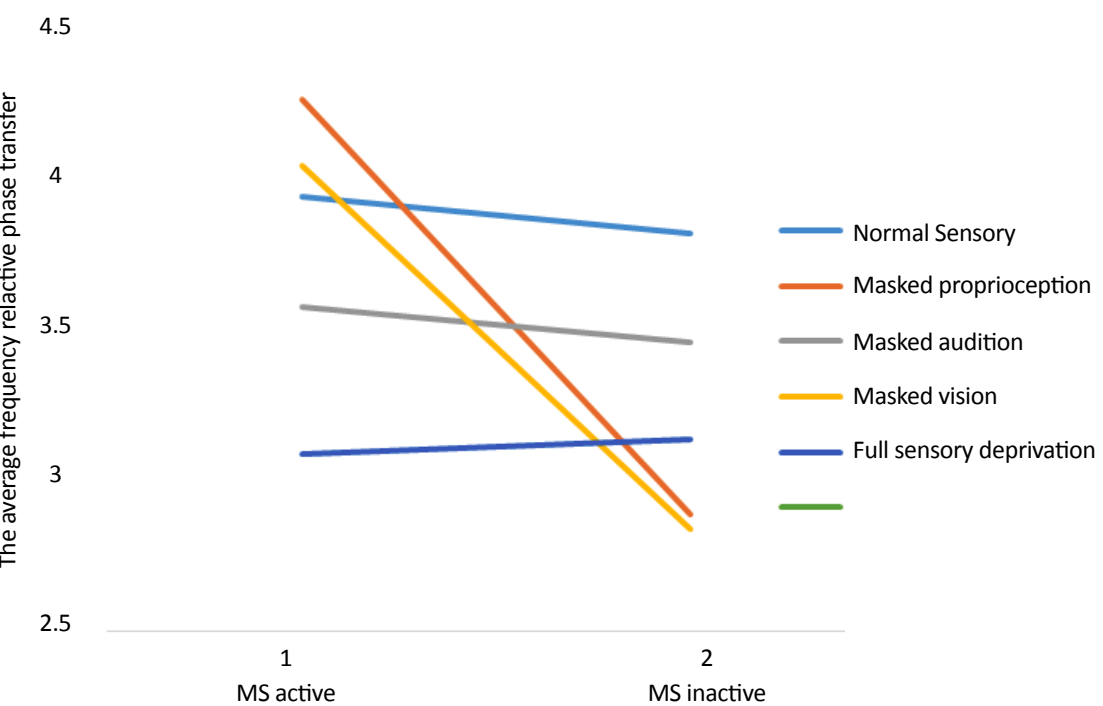

PHYSICAL TREA $\pitchfork$ MENTS

Figure 1. Histograms showing the average frequency relative phase transfer (AE scores) in two groups and five sensory conditions

tasks requiring them to produce in-phase or anti-phase movements with their wrists under different sensory conditions where the availability of visual, proprioceptive, and or auditory feedback was manipulated.

Results indicated that proprioception was more essential for the successful performance of the bimanual task than the other sensory modalities tested. Overall, this finding revealed that when proprioceptive input was manipulated (or masked) with tendon vibration, participants performed bimanual coordination tasks with higher mean relative phase error scores and poorer coordination consistency as compared to other conditions when proprioception was not manipulated. Stated differently, the reliance on proprioception in performance was evident not only during the performance of the "non-stable" anti-phase patterns but also in the "stable" in-phase patterns, suggesting that bimanual coupling appears to be controlled, for the main part, by proprioceptive information. These findings are largely supported by previous

Table 2. Results of ANOVA test to determine the effect of various senses in both active and inactive female patients with MS






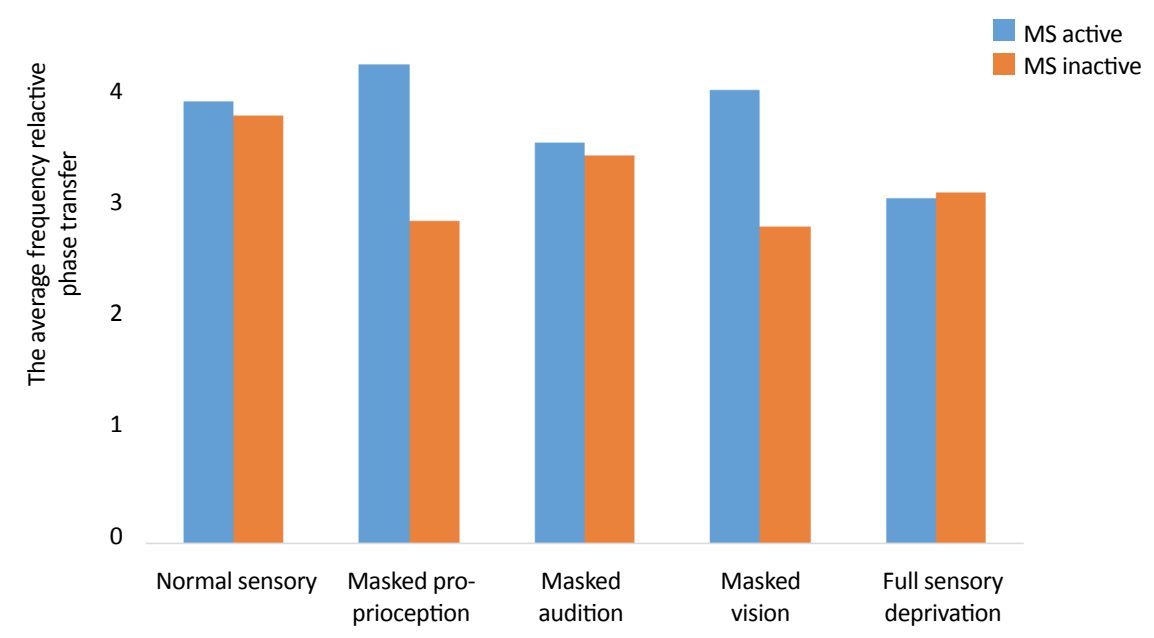

PHYSICAL TREA † MENTS

Figure 2. Histograms showing the average frequency relative phase transfer (AE scores) in the MS active and inactive groups in five different sensory conditions

studies where proprioception was either absent [23] or masked [17, 26, 34].

Our results agree with positive effects of exercise on proprioception. In Swanik [35] study, 6-week plyometric training (exercise that use muscle stretching and contraction for improving muscle function) improved proprioception of the shoulder in women swimmers. In Sharbatoghli [36] study, by using rhythmic exercises (rhythmic stabilization, tilt board) on lumbosacral area, a significant improvement was reported in proprioception. According to conducted research, exercise improves proprioception. The present study was in agreement with Swanik [35] and Sharbatoghli [36] studies, which reported that exercise therapy program can improve proprioception and reduce error angles (that is criteria for evaluating proprioception error) at least in healthy young women [37].

A possible mechanism to improve proprioception due to exercise is increasing attention. Attention is a neuropsychological process by which the central nervous system affects received information. Probably, proprioceptive training increases proprioception signs (first, at the conscious level and then at the automatic level) in the brain. Other possible mechanisms to explain the improvement in proprioception as a result of exercise, can be attributed to the activated pathway, increasing the number of synapses, and higher plasticity seen in the sensory area. However, these possible mechanisms in changing proprioception have not been justified yet. Studies have also shown that muscle spindle output can be increased voluntarily by changing the tone that eventually increase accuracy [38]. Myers et al. [39] also believe that the specific rehabilitation techniques are effective in improving motor sensory system, mechanical retrieval receptor afferents of the nervous system and facilitate the afferents aid as a compensatory mechanism that caused by defects in proprioception [39]. However, the discussed mechanism can only be seen in patients with lesions but not in normal subjects [37].

In this study, the complicated data from the wrist joints were altered by means of tendon vibration. It was shown that movement patterns with vibrations produce larger mean relative phase error than those without vibrations. Vibratory stimuli activate muscle spindles, through increase in their discharge levels. The extra stimulation of these receptors creates an artificial input and introduces a discordant source of afferent messages. Considering the convergence of different sources of proprioceptive information, whether from an external perturbation (vibration) or an active movement, one can hypothesize that the available afferent inflow is distorted and does not correspond to the actual situation at wrists. Interference prevails, leading to less accurate coordination patterns and an increase of relative phase errors. Our findings were, consistent with the findings reported in a study by Baldissera et al. and Serrien et al. [10, 17] indicating the need for attention. The present study also shows that the triceps and anterior deltoid muscle vibration lead to reducing the amplitude of the drawing circles. The vibration of the wrist flexor tendon destroys coordination between the two hands [10, 17, 34]. This result shows that proprioceptive information plays an essential role in the central nervous system for keeping posture [17]. The present study agrees with Grillo et al. [28] and Rocha et 
al. [40] research. They found that audition manipulation did not change the subjects' performance $[28,40]$.

Our findings were, nonetheless, in contrast to the findings of Grillo et al. [28]. This may be attributed to differences in procedure applied in two studies to deprive vision stimuli. First, in Grillo et al. [28] study combination of the vision and audition was not under control. Therefore findings from this study lack the ability to determine the extent by which participants integrate the two sensory modalities (i.e. vision and audition). Second, vision in Grillo et al. [28] study was manipulated by switching off the lights in the room, which may have allowed participants to use some visual input whereas in our study the occultation of vision was complete. Participants in our study were blindfolded, allowing complete preclusion of visual feedback. Where the sensory input from the hand was biased during ipsilateral hand and foot coordination at different levels of speed.

Our study observations also suggested that afferent information was elaborated differently during in-phase and anti-phase movements. For the in-phase movements (isodirectional movements), inferences are matched by a feedback mechanism automatically, whereas for antiphase movements (non-isodirectional movements), the afferent signals rely more strongly on a controlling process requiring abundant purposeful demands. It is important to note that Baldissera et al. [10] examined the effect of sensory bias on the coordination of ipsilateral hand and foot movements whereas the task/effectors tested in our study were bimanual coordination with the wrists. Nonetheless, trends in behavior that emerged for both studies were similar. Specifically, both studies showed that the influence of sensory bias on relative phase error was more pronounced during execution of the difficult tasks than the easy ones. Taken together, our study findings and that of Baldissera et al. [10] suggest that processing of sensory information in general and afferent input in particular are essential for successful performance of coordinated movements irrespective of the task and or effector combinations.

The availability of visual feedback also influenced the performance of subjects in the present study. This finding was in agreement with the observations reported in the studies of Cardoso de Oliveira and Barthelemy [16], showing that the presence of visual information enabled stable in-phase movements, meanwhile the stability of the anti-phase movements. The latter finding suggests that visual monitoring influences the production of both coordination modes in a different way. It might be interpreted as follows. During in-phase coordination, the extremities of both hands are in central vision and when the reversal occurs in a flexed position. This position may allow for an adequate calibration in terms of spatiotemporal dimensions. During anti-phase coordination, the bimanual phase offset never permits this optimal position because one hand will always be out of view [17]. In contrast to vision and proprioception the presence or absence of audition has no significant influence on performance. Our findings were in contrast to those of Ghez et al. [41] that used discrete auditory cues for audition feedback. This contrast was related to the method by which auditory feedback was presented to the participants. When we experimentally manipulated auditory feedback, for example by delaying audition, motor performance was significantly influenced. Yet, by obstructing task sound with headphone like in the study of Grillo et al. [28] and in the present study, coordination performance was not affected. These findings replicated those from previous research $[7,42]$. These findings can be explained based on ideomotor theories of action control [43]. The vital presumption of this method is that motor actions are cognitively indicated by their sensory impacts, i.e. by the codes of the impressive impacts that progressively follow certain motor actions. Consequently a motor action can solely be achieved by recollecting the codes of the sensory ramifications that normally come along with this action, and serve to mentally represent it. Put it in a different way, there is no other way to produce a motor action other than by anticipating its sensory ramifications.

The important implication of this approach is that all the constraints of motor control that we know of, such as complexity effects, stimulus-response compatibility, and or symmetry tendencies in bimanual coordination, do not arise because of inherent constraints in the structure and or functions of the motor system, but due to constraints in the representation of the perceptual differences of tobe-produced motor actions [44]. More studies should be conducted to further explore this hypothesis.

\section{Acknowledgements}

This research did not receive any specific grant from funding agencies in the public, commercial, or not-forprofit sectors. I would like to thank the Department of Motor Behavior and Sport Management, Faculty of Sport Sciences, Urmia University, for funding the present paper.

\section{Conflict of Interest}

The authors declared no conflicts of interest. 


\section{References}

[1] Sarlegna FR, Gauthier GM, Blouin J. Influence of feedback modality on sensorimotor adaptation: Contribution of visual, kinesthetic, and verbal cues. Journal of Motor Behavior. 2007; 39(4):247-58. doi: 10.3200/jmbr.39.4.247-258

[2] Bédard P, Proteau L. On the role of static and dynamic visual afferent information in goal-directed aiming movements. Experimental Brain Research. 2001; 138(4):419-31. doi $10.1007 / \mathrm{s} 002210100739$

[3] Saunders JA, Knill DC. Humans use continuous visual feedback from the hand to control both the direction and distance of pointing movements. Experimental Brain Research. 2005; 162(4):458-73. doi: 10.1007/s00221-004-2064-1

[4] Sober SJ, Sabes PN. Multisensory integration during motor planning. Journal of Neuroscience. 2003; 23(18):6982-92. PMID: 12904459

[5] Jackson G, Jackson S, Newport R, Harvey M. Co-ordination of bimanual movements in a centrally deafferented patient executing open loop reach-to-grasp movements. Acta Psychologica. 2002; 110(2-3):231-46. doi: 10.1016/s00016918(02)00035-5

[6] Carson RG, Thomas J, Summers JJ, Walters \& Andras Semjen MR. The dynamics of bimanual circle drawing. The Quarterly Journal of Experimental Psychology Section A. 1997; 50(3):664-83. doi: 10.1080/713755721

[7] Kelso, JA. Phase transitions and critical behavior in human bimanual coordination. American Journal of Psychology. 1984; 246(6 Pt 2):R1000-4. PMID: 6742155

[8] Salter JE, Wishart LR, Lee TD, Simon D. Perceptual and motor contributions to bimanual coordination. Neuroscience Letters. 2004; 363(2):102-7. doi: 10.1016/j.neulet.2004.03.071

[9] Swinnen SP, Jardin K, Meulenbroek R, Dounskaia N, Den Brandt MHV. Egocentric and allocentric constraints in the expression of patterns of interlimb coordination. Journal of Cognitive Neuroscience. 1997; 9(3):348-77. doi: 10.1162/ jocn.1997.9.3.348

[10] Baldissera F, Cavallari P, Marini G, Tassone G. Differential control of in-phase and anti-phase coupling of rhythmic movements of ipsilateral hand and foot. Experimental Brain Research. 1991; 83(2): 375-380. doi: 10.1007/bf00231161

[11] Li Y, Levin O, Forner-Cordero A, Ronsse R, Swinnen SP. Coordination of complex bimanual multijoint movements under increasing cycling frequencies: The prevalence of mirror-image and translational symmetry. Acta Psychologica. 2009; 130(3):183-95. doi: 10.1016/j.actpsy.2008.12.003

[12] Swinnen SP, Dounskaia N, Verschueren S, Serrien DJ, Daelman A. Relative phase destabilization during interlimb coordination: The disruptive role of kinesthetic afferences induced by passive movement. Experimental Brain Research. 1990; 105(3):439-454. doi: 10.1007/bf00233044

[13] Kelso JA, Jeka JJ. Symmetry breaking dynamics of human multilimb coordination. Journal of Experimental Psychology: Human Perception and Performance. 1992; 18(3):645-68 doi: 10.1037/0096-1523.18.3.645

[14] Li Y, Levin O, Carson RG, Swinnen SP. Bimanual coordination: Constraints imposed by the relative timing of homolo- gous muscle activation. Experimental Brain Research. 2004; 156(1):27-38. doi: 10.1007/s00221-003-1762-4

[15] Sigrist R, Rauter G, Riener R, Wolf P. Augmented visual, auditory, haptic, and multimodal feedback in motor learning: A review. Psychonomic Bulletin \& Review. 2012; 20(1):21-53. doi: 10.3758/s13423-012-0333-8

[16] Cardoso de Oliveira S, Barthelemy S. Visual feedback reduces bimanual coupling of movement amplitudes, but not of directions. Experimental Brain Research. 2004; 162(1):7888. doi: $10.1007 /$ s00221-004-2107-7

[17] Serrien DJ, Teasdale N, Bard C, Fleury M. Age-related differences in the integration of sensory information during the execution of a bimanual coordination task. Journal of Motor Behavior. 1996; 28(4):337-47. doi: 10.1080/00222895.1996.10544603

[18] Bernier PM, Chua R, Franks IM. Is proprioception calibrated during visually guided movements? Experimental Brain Research. 2005; 167(2):292-6. doi: 10.1007/s00221-005-0063-5

[19] Bourdin C, Gauthier G, Blouin J, Vercher JL. Visual feedback of the moving arm allows complete adaptation of pointing movements to centrifugal and Coriolis forces in human subjects. Neuroscience Letters. 2001; 301(1):25-8. doi: 10.1016/s0304-3940(01)01584-1

[20] Scheidt RA. Interaction of visual and proprioceptive feedback during adaptation of human reaching movements. Journal of Neurophysiology. 2005; 93(6):3200-13. doi: 10.1152/jn.00947.2004

[21] Cordo P, Bevan L, Gurfinkel V, Carlton L, Carlton M Kerr G. Proprioceptive coordination of discrete movement sequences: mechanism and generality. Canadian Journal of Physiology and Pharmacology. 1995; 73(2):305-15. doi: 10.1139/y95-041

[22] Norouzi E, Farsi A, Vaezmousavi M. Effects of proprioceptive and visual disturbance on inphase and anti-phase hand performance. Physical Treatments-Specific Physical Therapy Journal. 2015; 5(1):41-48.

[23] Sainburg RL, Poizner H, Ghez C. Loss of proprioception produces deficits in interjoint coordination. Journal of Neurophysiology. 1993; 70(5):2136-47. PMID: 8294975

[24] Bonnard M, Pailhous J. Contribution of proprioceptive information to preferred versus constrained space-time behavior in rhythmical movements. Experimental Brain Research. 1999; 128(4):568-72. doi: 10.1007/s002210050883

[25] Ghez C, Sainburg R. Proprioceptive control of interjoint coordination. Canadian Journal of Physiology and Pharmacology. 1995; 73(2):273-84. doi: 10.1139/y95-038

[26] Verschueren SMP, Swinnen SP, Cordo PJ, Dounskaia NV. Proprioceptive control of multijoint movement: Unimanual circle drawing. Experimental Brain Research. 1999; 127(2):171-81. doi: 10.1007/s002210050787

[27] Hermann T, Höner O, Ritter H. AcouMotion - an interactive sonification system for acoustic motion control. Gesture in Human-Computer Interaction and Simulation. 2006; 31223. doi: $10.1007 / 1167881635$

[28] Grillo EU, Almeida Q, Lee TD, Abbott KV. Do vision and audition influence bimanual timing coordination for in-phase and anti-phase patterns in a linear slide task? 
Open Sports Sciences Journal. 2010; 3(1):105-10. doi: 10.2174/1875399x01003010105

[29] Schmidt RA, Wrisberg RA. Motor learning and performance: Situation-based learning approach. [A. Naseri Persian trans]. $4^{\text {th }}$ edition. Tehran: Elm va Harkat Publishers; 2008.

[30] Yektamaram SA. Nazmdeh K. [Timely rehabilitation in patients with multiple sclerosis (Persian)]. Tehran: State Welfare Organization of Iran; 2009.

[31] Holland NJ, Halper J. Multiple sclerosis: A self care guide to wellness. New York: Demos Medical Publishing; 2005.

[32] Oldfield RC. The assessment and analysis of handedness: The Edinburgh inventory. Neuropsychologia. 1971; 9(1):97113. doi: 10.1016/0028-3932(71)90067-4

[33] Naito E, Ehrsson HH, Geyer S, Zilles K, Roland PE. Illusory arm movements activate cortical motor areas: A positron emission tomography study. The Journal of Neuroscience. 1999; 19(14):6134-44. PMID: 10407049

[34] Steyvers M, Verschueren S, Levin O, Ouamer M, Swinnen S. Proprioceptive control of cyclical bimanual forearm movements across different movement frequencies as revealed by means of tendon vibration. Experimental Brain Research. 2001; 140(3):326-34. doi: 10.1007/s002210100819.

[35] Swanik KA, Lephart SM, Swanik CB, Lephart SP, Stone $\mathrm{DA}, \mathrm{Fu} \mathrm{FH}$. The effects of shoulder plyometric training on proprioception and selected muscle performance characteristics. Journal of Shoulder and Elbow Surgery. 2002; 11(6):579-86. doi: 10.1067/mse.2002.127303

[36] Sharbatoghli R. [Comparative study of the effects of two proprioception training methods (rhythmic stabilisation, tilt board) on lumbosacral proprioception (Persian)] [MSc. thesis]. Tehran: Shahid Beheshti University of Medical Sciences; 2001.

[37] Paci M. Structure, physiopathology and rehabilitation of the sacroiliac joint. Europa Medicophysica. 1999; 35(4):20718.

[38] Ashton-Miller JA, Wojtys EM, Huston LJ, Fry-Welch D. Can proprioception really be improved by exercises? Knee Surgery, Sports Traumatology, Arthroscopy. 2001; 9(3):12836. doi: $10.1007 / \mathrm{s} 001670100208$

[39] Myers JB, Wassinger CA, Lephart SM. Sensorimotor contribution to shoulder stability: Effect of injury and rehabilitation. Manual Therapy. 2006; 11(3):197-201. doi: 10.1016/j. math.2006.04.002

[40] Roche R, Wilms-Floet AM, Clark JE, Whitall J. Auditory and visual information do not affect self-paced bilateral finger tapping in children with DCD. Human Movement Science. 2011; 30(3):658-71. doi: 10.1016/j.humov.2010.11.008

[41] Ghez C, Rikakia T, Cook PR. An auditory system for aiding inter joint coordination. Paper presented at: The International Conference on Auditory Display; 2000 April 2-5; Atlanta, USA.

[42] Scholz, JP, Kelso, JAS. Intentional switching between patterns of bimanual coordination depends on the intrinsic dynamics of the patterns. Journal of Motor Behavior. 1990; 22(1):98-124. doi: 10.1080/00222895.1990.10735504
[43] Hommel B, Müsseler J, Aschersleben G, Prinz W. The Theory of Event Coding (TEC): A framework for perception and action planning. Behavioral and Brain Sciences. 2001; 24(5):849-78. doi: 10.1017/s0140525x01000103

[44] Janczyk M, Skirde S, Weigelt M, Kunde W. Visual and tactile action effects determine bimanual coordination performance. Human Movement Science. 2009; 28(4):437-49. doi: 10.1016/j.humov.2009.02.006 
